\title{
Formal and substantial Internet information skills: The role of socio- demographic differences on the possession of different components of digital literacy
}

\author{
Marco Gui
}

\begin{abstract}
The literature about digital inequality has pointed out the role of so-called "digital skills" in contributing to a full exploitation of the opportunities of the Web for individuals. Research has started to measure the differences in online skills on a socio-demographic base, finding relevant disparities. Since different components of digital skills have been described in theory, it is not clear which of them are influenced by specific social variables and which are not. This study goes a step further in the analysis of "digital skills," concentrating on two different components of them: "formal information skills" and "substantial information skills." Complex search tasks were assigned to a quota sample made up of young people in northern Italy, divided by gender and education level. The results show that when other important variables associated with digital skills (age, experience with the Web, availability of hardware and software) are kept constant differences in gender and education have an influence on substantial information skills, but not on formal ones. This provides evidence for the assumption that a substantial part of digital skills represent a socially relevant factor for digital inequality, even when education and the spread of new media have standardized the level of formal skills.
\end{abstract}

\section{Introduction: Inequalities in Internet use}

What kind of ability is it which quickly and correctly finds different kinds of information on the Internet when it is needed? Is it a manual-technical ability whose measurement is to be considered by the same standards as videogame playing ability? Or is it an ability which can influence the cultural and social life of an individual? 
In the sociological tradition, differences in using media and accessing information among individuals has very rarely been considered a relevant source of inequality. Even in the specific field of mass media sociology (with the relevant exception of the knowledge gap hypothesis [1]), the abilities to use and exploit media have not been linked directly to social inequality. The advent of digital media has changed this situation and opened a wide debate about inequalities derived from different possibilities of exploiting new informational opportunities.

The use of the Internet differently from that of mass media (like radio and TV) is based very much on personal resources. The activities of information selection, evaluation, filtering, recombination — which were once carried out by media professionals — are shifting back to the individual. As such, individuals have more freedom but also find themselves alone in building their own agendas, content and strategies relative to information consumption.

The digitalization process has been the means for getting media consumption out of the Fordist era, offering possibilities of personalization and customization already present in other areas of consumption. However, in the case of consumption of information, differentiation can have larger and less predictable social consequences impacting more profoundly on the cultural, professional and social lives of individuals.

Initially worries about the "digital divide" were focused on the growing disadvantages experienced by those without a connection to the Internet. However, since 2000 much literature has been devoted to enlarging the concept of the "digital divide" into a broader one of "digital inequality" (DiMaggio, et al., 2004; Hargittai, 2002, 2004a; van Dijk and Hacker, 2003; van Dijk, 2005).

Apart from the problems based on a sheer lack of connection, notions of "digital inequality" examine the effects of differences in the use of the Internet among people who already have physical access to the computers and digital networks. As new media spreads and becomes more pervasive in our daily lives, the real digital divide seems to be based on what people do with media, more than on technologies and connectivity available to them. As van Dijk [2] effectively states, it seems that the problem usually called "digital divide" is "deepening where it has stopped widening", that is when the number of people without access to the Internet diminish, a new and more profound divide can be created because of different levels of ability and opportunities to exploit a very complex medium with diverse and abundant content.

Many authors have proposed various descriptions of the causes of "digital inequality". Particularly relevant among the components of digital differences are: autonomy of use (DiMaggio, et al., 2004); motivation (van Dijk, 2005); support from social networks (DiMaggio, et al., 2004); and, types and impact of use (Bonfadelli, 2002; van Dijk, 2005; Liff and Shepherd, 2005). This paper 
examines digital skills, described by a number of other researchers (van Dijk and Hacker, 2003; van Dijk, 2005; Warschauer, 2002; Hargittai, 2002, 2004a, 2004b; Hargittai and Shafer, 2006; Steyaert, 2000; Norris, 2001).

To date, there have been few studies which try to empirically observe "digital skills". Van Dijk (2005) notes that "despite a growing literature on Web-site usability issues (much of it from library science and social informatics), we know little about how and why skill is related to personal characteristics". Most of the works focus on psychological differences, pointing out the importance of personal characteristics such as field dependency/independency (Wang, et al., 2000; Palmquist and Kim, 2000; Kim and Allen, 2001; Juvina and van Oostendorp, 2004); gender (Ford, et al., 2001); experience (Lazonder, et al., 2000); and, locus of control, motivation and interest (Juvina and van Oostendorp, 2006).

Even scarcer are the studies which take socio-demographic characteristics into consideration. Hargittai (2002) reported the first research of this kind based on a random sample of residents in a socially heterogeneous New Jersey county. The subjects were tested while they tried to locate several kinds of material online. Hargittai defined the concept of skills as the ability to complete a task and the amount of time spent for completing it. The author found large differences among the subjects, influenced by age, education and prior experience with the technology. Hargittai did not find significant differences based on gender.

But which of the different components of digital skills are we talking about? Here I argue that social variables have a different influence on digital skills depending on which component of them we test. The research reported here makes a first important distinction: on the basis of van Dijk's model (van Dijk, 2005), I try to observe "formal" and "substantial information skills" separately.

In this paper research results attempt to differentiate and separately measure formal and substantial information skills in retrieving information on the Internet. This is done in order to understand if the two components of skills are influenced differently by social characteristics such as gender and education capital while maintaining other variables constant. The results suggest that formal and substantial skills have very different social implications.

\section{Formal and substantial information skills}

Many authors have tried to define what can be assumed by the term "digital skills". This very complex concept is formed by different problems, and is connected to what is believed to be a real new form of literacy (Warschauer, 2002). Many scholars who analyzed the concept of digital skills 
distinguish between two main components: "technical/operational skills", which are needed to operate computers and basic software, and "informational skills", those needed to navigate, select, evaluate and re-use information. The main feature of this second component, "information skills", is to be formed not only by skills directly connected to the use of new media, but also by skills which were present in traditional literacy and become even more important in the era of digital media.

Van Dijk (2005) proposes what is probably the most interesting model so far to distinguish between different parts of digital skills.

- Operational skills

- Informational skills

○ formal

- substantial

- Strategic skills

The first component, that of "operational skills", regards the ability needed to operate computers and Internet connections with their basic applications. Its standardization is well represented by the seven modules of the European Computer Driving License (ECDL). Individuals in many western countries demonstrate considerable differences in operational skills [3]. However, differences in this component seem to be resolved in the long term with formal education (parallel to literacy) and especially with the natural spread of technologies in the population. This makes differences in operational skills more a contingent rather than a structural social problem.

The third component, strategic skills, concerns the abilities to determine the general goals of media use in a universe of information overload and disorganization. Strategic skills are "defined as the capacities to use computer and network sources as the means for particular goals and for the general goal of improving one's position in society". [4] As van Dijk argues, such a goal-oriented attitude is more important with new media and in a "network society" than it was in the past.

We focus in this paper on the second component: "information skills". Van Dijk defines them as the skills to search, select and process information in computer and network sources. As strategic skills are very hard to measure, most research on Internet skills implicitly or explicitly focuses on information skills. In fact, they can be observed when subjects need to solve complex information retrieval activities, when operational skills are constant and when the task is given [5]. 
Van Dijk proposes an interesting sub-distinction of information skills. "Formal information skills are the abilities to understand and work with the formal characteristics of a particular medium ... Substantial information skills are the abilities to find, select, process and evaluate information in specific sources following particular questions.” [] $]$

In other words, formal information skills are related to the forms in which contents are organized inside new media, such as the Internet. For example, these competences are needed to move rapidly and efficiently about hyperlinks, to know and make proper use of features such as bookmarks, to understand the structure of an URL and to be able to locate the home page starting from a complex address. However, more profound skills are needed to use the structure with proper content.

Substantial information skills are needed for this deeper digital literacy component. They are needed to search, select, process and evaluate information as it is presented on the Internet and new media. Examples of activities which need substantial information skills are: evaluate the nature of a site (commercial, personal home page, governmental, lobby), evaluate the reliability of the contents of a site, be able to select the most useful links in a search engine page results, simplify complex research activities, produce good queries for search engines [7]

In the practice of navigation, formal and substantial information skills are interrelated. For example, an individual can be good at evaluating the content of a site (substantial skill) if he knows how to efficiently use links and combo menus in order to easily navigate it and process its contents (formal skills).

However, these two components of skills are also conceptually very different. In the analysis of Internet information retrieval skills (as said before, mostly focused on information skills) identified differences were not divided between these two levels. We know that social characteristics such as age, gender, education can have an influence on information retrieval skills on the Internet (Hargittai, 2002, 2004a, 2004b; Dietrichson, 2001). But we do not know what specific kind of skills can be most influenced by these social variables. By knowing this, it will be possible to understand where the social relevance of the differences of digital skills lies and to better focus policies and solutions.

The goal of this study is to investigate if there is a different influence by gender and education capital on formal and substantial information skills, when other important variables (age, experience, conditions of access) are kept constant. We do this by separately measuring the performances of people in tasks which are related to one or the other component. 
My hypothesis is that gender and education influence Web information retrieval performances, even among people with the same physical access conditions and the same age and length of experience in using the Internet. In particular, among users with these characteristics, gender and education capital influence performance in information retrieval only for substantial information skills and not formal information skills (as van Dijk, 2005, defines them).

This hypothesis is derived from preliminary and non-scientific observations of information retrieval activities. The hypothesis can also be inferred theoretically by van Dijk's model: the higher the level in the hierarchy of digital skills, the less abilities depend on the mere familiarity with the media and are linked to more profound skills. Gender and education are two important sociological variables in the field of new media use. The second has been considered by many scholars as a relevant predictor of selective use, good acceptance and storage of information, both in "old media" (Tichenor, et al., 1970) and in new media (van Dijk, 2000, 2005, 2006; DiMaggio, et al., 2004; Hargittai, 2002, 2004a, 2004b; Bonfadelli, 2002). As for gender, it is also an important variable in the field of new media studies. Some research (Hargittai and Shafer, 2006; Liff and Shepherd, 2005) considered it more relevant with respect to self-evaluation of skills (it is clear that females tend to underrate themselves) than to real performances. Hargittai and Shafer (2006) found that females are equal to males when it comes to actual performance. Will this finding be confirmed after a separate analysis of formal and substantial information skills?

To separately test the two components, I have used an exploratory methodology. Navigation performances inside closed hypertexts (especially inside a single site) have been used as indicators of formal information skills. In closed hypertext navigation tasks, users only have to locate information in a given information structure; they follow links, use combo menus, maps and internal search engines. In this activity, formal information skills are extremely prominent since finding an information is only an activity of location.

Navigation performances involving the entire Web have been used as indicators of substantial information skills. To retrieve information in the open Web, not only formal information skills are needed but mostly substantial information skills (especially in the first parts of the session). To be successful, users have to create appropriate queries on search engines, select results efficiently on the basis of their quality, and know where to look for resources.

Although in open Web navigation also some formal information skills are needed, performances in these types of activities can be used to measure the impact of substantial information skills in comparison with performances in closed hypertext tasks, where mostly formal skills are needed. 
If my hypothesis is true, we expect to find different effects of gender and education capital in closed hypertext and open Web tasks. In the former differences should be negligible while in the latter differences should be relevant.

This research is significant to our understanding of digital inequalities. It is possible that with the spread of the Internet and new media to the majority of the population operational and formal information skills will be standardized by use and by more formal training in schools.

If the hypothesis is true, it means that even among people of the same age, with the same technical tools and experience with the Internet, social characteristics will influence those skills needed for a complete exploitation of the Internet's potential. By understanding if substantial information skills continue to be influenced by social characteristics, even when formal information skills are constant, we can more specifically examine which skills are relevant to inequalities in the long term.

Hence, these results are relevant to educational planning and for policies aimed at reducing digital inequalities. If my hypothesis is true, schools must devote a special effort to substantial information skills instead of operational skills.

\section{Research}

A growing body of empirical research has appeared examining how individuals search for information on the Web. Apart from marketing studies (the results of which are often not accessible to the public), much of this literature comes from the field of psychology. Many studies observed performances in information retrieval activities as an effect of psychological variables, such as "field dependency" or "field independency." [ㅇ]

Some studies on information retrieval performances on the Web were also conducted in the computer sciences. The main goal for computer scientists is to develop tools to help people navigate by observing them in real time (see Juvina and van Oostendoorp, 2004, Juvina and Herder, 2004). Few studies use social characteristics as independent variables (Hargittai, 2002, 2004a, 2004b; Hargittai and Shafer, 2006; van Dijk, et al., 2000; de Haan, 2003; Dietrichson, 2001; Gui, 2006). In particular, Hargittai (2002) presented one of the first empirical observations about the influence of social positioning on digital literacy. She tested a sample of 54 demographically diverse individuals during activities of information retrieval on the Internet. With a mixed methodology (in-person observation and analysis of audio-video files generated by screen capture applications), she found a wide variety of differences both in the ability to solve the tasks assigned and in the time to complete 
them. The study did not find significant gender differences, while age and education emerged as highly significant. In another study (Hargittai and Shafer, 2006) it was found that the self-assessed skill of women was significantly lower than that of men, but there are no significant differences when it comes to applied abilities.

From the studies of van Dijk (2000) and de Haan (2003) it can be assumed that "those having a high level of traditional literacy also possess a high level of digital information skills [...] These skills appear to be more important for computer and Internet use than technical know how and the capacity to deal with numerical data." (van Dijk, 2006) Research also suggests that people learn information skills in practice, by trial and error, less than in formal educational settings (de Haan and Huysmans, 2002; van Dijk, 2005, cited in van Dijk, 2006).

What kind of digital information skills are these results referable to? Do these variables influence all parts of information skills, or are they connected to some of them in particular?

The research presented here attempts to go a step further and separately observe formal and substantial Internet information skills. Data were collected with a navigation-tracking methodology for the evaluation of performances of information retrieval on the Web. Differently from many studies of Web searching which use personal observation or audio-video recording, the performances of the subjects were measured thanks to data generated automatically by tracking software during each session of navigation. A shareware application (Scone), downloadable at http://www.scone.de, was used to register information about the path and content viewed by subjects during the completion of the tasks assigned.

To focus on the role of formal and substantial information skills, a sample was formed in which operational skills could be considered constant. The subjects, therefore, have the same age (25-35 years old), the same availability of technology and spend the same amount of time on the Internet per week. Furthermore, they all use the Internet both at home and at work (in this last case, they all have broadband access). The subjects, instead, differ for two important social characteristics: gender and education capital [9]. The quotas were obtained by crossing dichotomous states of these two variables:

20 males with high education capital

20 males with medium education capital

20 females with high education capital

20 females with medium education capital 
The subjects were recruited by the interviewer in universities and firms of northern Italy. Since they were not offered any monetary reward for participation, a laptop computer with the tracking software installed was used to test the subjects in their own offices or workplaces in order to minimize their efforts.

Before the test subjects were also asked to answer a quick questionnaire to make sure they entered in one of the quotas and to learn some contextual information about user habits in Internet. As in Hargittai (2002), the ability to find information on the Web was measured by giving respondents tasks to perform online. Subjects had to find the answers to the following four questions in this order:

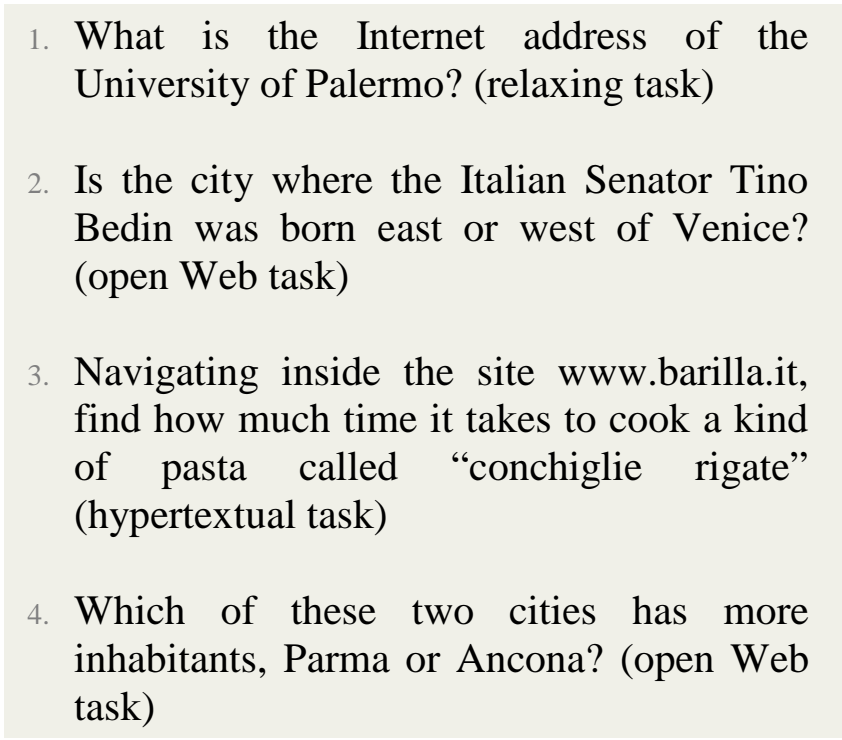

After an analysis of the tasks used in literature for online retrieval research (Kim and Allen, 2001; Hargittai, 2002; Wang, et al., 2000; Palmquist and Kim, 2000; White and Iivonen, 1999, 2001), the tasks were chosen to be: potentially useful in a particular moment in life for all the subjects; of a medium difficulty (to properly differentiate the performances); non-domain specific (if someone already knew the answer, he had to tell the interviewer and his data were not analyzed).

An interface would present the questionnaire to the subjects and after that each task would appear followed by another when the answer to the previous one was given. As in Hargittai (2002) skill was defined as the success of the search and time spent in completing it. 


\section{Differences in formal and substantial information skills}

Results have been analyzed for tasks 2, 3 and 4. Task 1 only had the role of a preliminary familiarization activity. Its low level of difficulty also had the role of favouring a positive emotional attitude, useful for a proper completion of the test.

In the table below, the general performance of each subgroup of the sample are presented. It is possible to see the average time spent in each subgroup to find correct answers, the number of total wrong answers given by each subgroup, and the standard deviation of the single times used for completion (of correct answers) in the subgroups.

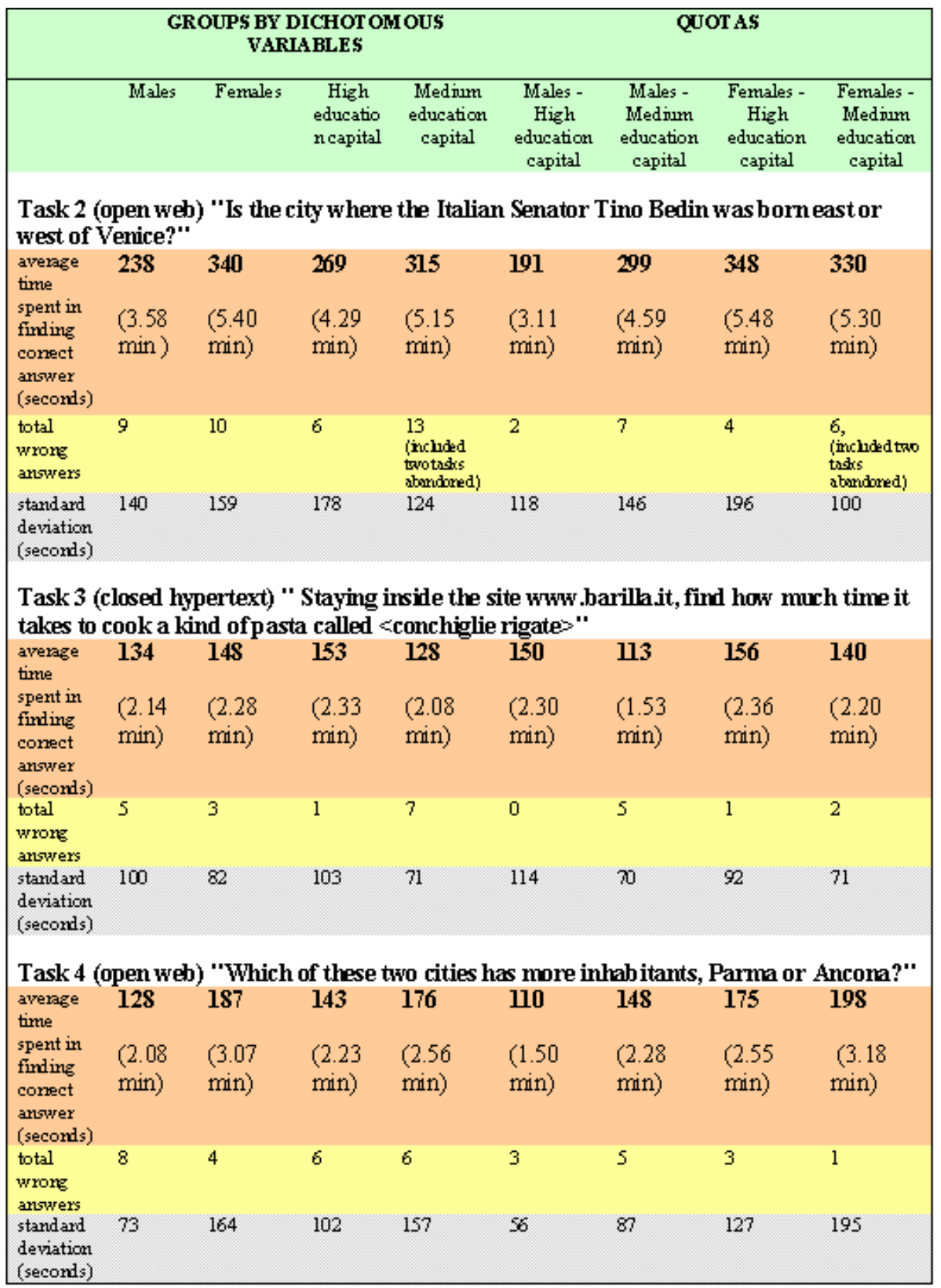


In task 2, gender and education capital (or e.c.) present some influence: the average time used by males and females has a difference of 1 minute and 42 seconds. Medium e.c subjects are a bit slower than their counterparts. But the combined effect of the two variables shows a relevant influence. The quota formed by males with high e.c is by far the quickest and most correct group. Furthermore, a low value of standard deviation suggests an internal homogeneity in the possession of high skills in this quota. This last group presents the only two abandoned tasks of the experiment. It is to be noted that the possibility to abandon was not even hypothesized by the interviewer. The worst average time is that of females with high e.c, although a very high standard deviation shows that this quota has the highest internal differentiation. The difference between the first and the last quota is 2 minutes and 37 seconds.

In task 3, there are no relevant differences regarding times, considering both variable subgroups and quotas The speed hierarchy is very different. Males with medium e.c produce more errors than the other male group. Standard deviation values are lower and less diverse than in the previous task. Errors are more frequent in the medium e.c quotas, especially among males. The difference between the first and the last quota is 43 seconds, which is very low although the task was judged as difficult by the subjects. It seems that in this task the difficulty creates problems for all quotas in a similar manner.

In task 4, the situation returns to the trend of task 2, although with less pronounced differences, probably due to the lower difficulty of the task. Males are faster than females by a minute. Males with high e.c are better than the other quotas and show a very low standard deviation. After them, as in task 2, there are males with medium e.c, then females with high and with medium e.c. The difference between the first and the last quota is 1 minute and 28 seconds.

In the two tasks to be solved by navigating the entire Web (task 2 and task 4) relative positions of the quotas appear similar. In both the tasks males with high e.c perform much better than the others. Their leading gap on the second quota (in both cases males with high e.c.) is greater than those between the other three quotas. In task 2, the gap is more than double the gap between the others. Furthermore, in both tasks males with high e.c. have low standard deviation levels. It seems that their advantage is shared homogeneously and similarly in the two tasks. Also Males with medium e.c behave similarly in the two tasks. They are second in the speed hierarchy and they show the highest number of errors and low standard deviation values. In task 3 none of these similarities can be seen.

Females are in the last two positions in both task 2 and task 4, but in task 3 they are second and last. In task 2 and 4 they show the highest values of standard deviation, suggesting that skill conditions are more different among females. But in task 3 they have low values of standard deviation. 
In task 2 and 4 the different level of difficulty (task 2 was judged as more difficult than 4 ) does not seem to influence the relative position of the quotas. In task 3 (also judged as difficult) the situation is completely different and none of the regularities highlighted emerge. The influence of e.c variable is opposite and the hierarchy is different. Males with medium e.c are the fastest, then females with medium capital, and finally the two quotas with high capital (but, as said, the range of times is not so relevant).

Furthermore, it is to be noted that females present much lower standard deviation values than in the other two tasks. Instead, males with high e.c have the highest value while in the other two tasks they were among the lowest. Considering time, errors and standard deviation it is possible to say that with the two open Web tasks important regularities emerge. Instead, the hypertextual task does not match with this scheme at all.

Results show that in the two open Web tasks (2 and 4) gender and e.c influence performances, both in time and errors. Being male and having a high education ends up in significantly better performances, both in time and number of errors. Furthermore, in both Web tasks males with high e.c have low standard deviation values, showing that the conditions of advantage are shared in the quota, while - among females - conditions vary more widely. These differences are not relevant in task 3 (close hypertext task) where the values do not differ among the groups and quotas. In task 3 , based on hypertextual navigation only, the hierarchy of the quotas based on efficiency is completely different and males with high e.c do not show any significant advantage.

The results are consistent with the initial hypothesis that gender and e.c have an influence on substantial information skills but not on formal information skills.

But what is there about specific substantial information skills which can explain these differences to find content online in open Web tasks? To offer a deeper understanding, the tracking of a subsample of the ten best and worst task navigations was analyzed. The software used for tracking can also offer the succession of visited pages with the time spent in each of them. It is possible to give some qualitative conclusions about the main characteristics which differentiate the navigation from the best and the worst subjects. In particular it can be noted that good performances are obtained because of these skills:

Production of queries with high probabilities of being contained in sites relevant for the research;

Proper use of advanced research tools, but not using 
them when it is not necessary (quotation marks, Google "cache copy," advanced research);

Ability to select relevant links in the results page of search engines, and once followed to quickly evaluate the path if it is promising;

Knowledge and familiarity with specific resources on the Web (geographical map sites, Google images, site of the Senate etc.) where the possibility of retrieving specific relevant information is assumed.

These skills are not referable to the knowledge of the formal structure of the Internet and new media in general. Since these skills are different also in people with the same age, experience with the Web, type of connection, they emerge as a first description of the substantial part of digital literacy. From a long-term perspective they are among the most socially relevant parts of digital inequality.

\section{Conclusion}

Digital skills are complex. The use of new media is more diversified than that of any other media in history, and it seems to be developing into a real new form of literacy. In this paper we focused on two components of it: formal and substantial information skills, as described by van Dijk (2005). We analyzed these skills empirically looking at the specific activity of information retrieval on the Web.

We have data supporting the initial hypothesis: gender and education capital influence performances in open Web tasks but do not seem to do the same with hypertextual navigation tasks.

We have considered performances in hypertestual tasks as indicators of formal information skills, and performances in open Web tasks as indicators of substantial information skills. By this operationalization, we show that when operational skills are constant gender and education capital can still influence the level of skills, but only for what concerns substantial information skills.

We suggest that the influence of sociodemographic variables such as gender and education on information retrieval abilities depend on the nature of the task. We are now able to make a distinction between open Web and closed hypertext tasks, which seems to be relevant. The first kind of tasks are capable of producing differences based on gender and education capital, the second ones are not. We can now say that substantial information skills are more influenced by 
social characteristics of subjects with the same age, Internet experience and physical access, while formal information skills can be thought as not significantly different in samples with these features.

The consequence is that in the world of a "second-level digital divide", as Hargittai (2002) defines it, substantial information skills, and not formal skills, seem to be more socially relevant on a longterm perspective. It is clear that some of the differences of digital skills can be seen as a contingent problem, others as more structural. The first ones will probably standardize since the spread of technology will provide the majority of people with operational and formal skills. Among the second ones, there are with no doubt substantial information skills (and probably strategic skills which still have to be measured empirically).

Educational policies that aim to reduce inequalities in the use of information technologies will need to re-consider their strategies. Schools and teachers, even if they are often much less skilled than their own pupils in terms of operational and formal skills, could be crucial in providing to students more substantial skills.

Notes

1. The "knowledge gap" hypothesis (Tichenor, et al., 1970) states that people with higher socioeconomic status have better capabilities to acquire and process media information. The consequence is that the more information provided by the media on a certain subject, the more diverse is the level of knowledge about that topic among different groups in society.

2. Van Dijk, 2005, p. 2.

3. See Park, 2002; de Haan, 2003, quoted in van Dijk, 2006.

4. Van Dijk, 2005, p. 88 .

5. The individual decision process that regards the goals of the use of new media is covered by strategic skills.

6. Van Dijk, 2005, p. 81 .

7. Van Dijk (2005) examines seven formal information skills (pp. 82-86): "Getting to know and to control the file structure of a computer and the Internet"; "Getting to know and to control the information structure of the Internet in general and a website in particular"; "Getting to know and to control the hyper-link structure of the Internet"; "Getting to know and to control the lay-out and design of multimedia screens"; "Learning to handle the fragmented 
nature of computer and network sources"; "Learning to handle the continually changing contents of computer and Internet information sources"; "Ability to read and write English".

He identifies the following six substantial information skills: "Learning to search information"; "Learning to select information continually (not only in search operations)"; "Learning to edit information oneself”; "Being able to apply a quality assessment of information sources in computer files and on the Internet"; "Being able to combine information from an increasing number of media, channels and individual sources"; "Being able to derive associations and generalizations from specific pieces of information”.

8. For a review see Chen and Macredie, 2002.

9. This variable regards the education level of the subject and of his or her parents. Two states of this variable are considered in this research: high education capital (when the subjects and at least one of his/her parents have a university degree), medium education capital (when the subjects and his/her parents do not have a university degree). In this last case, the subjects have a high school diploma.

\section{References}

H. Bonfadelli, 2002. "The Internet and knowledge gaps: A theoretical and empirical investigation," European Journal of Communication, volume 17, number 1, pp. 65-84.

S. Chen and R. Macredie, 2002. "Cognitive styles and hypermedia navigation. Development of a learning model," Journal of the American Society for Information Science and Technology, volume 53, number 1, pp. 3-15.

P. DiMaggio, E. Hargittai, C. Celeste, and S. Shafer, 2004. "From unequal access to differentiated use: A literature review and agenda for research on digital inequality," In: K. Neckerman (editor). Social inequality. New York: Russell Sage Foundation, pp. 355-400.

J. de Haan, 2003. "IT and social inequality in the Netherlands," IT and Society, volume 1, number 4, pp. 27-45.

J. de Haan and F. Huysmans, 2002. "Differences in time use between Internet users and nonusers in the Netherlands," IT and Society, volume 1, number 2, pp. 67-85.

A. Dietrichson, 2001. "Digital literacy: How to measure browsing behaviour," $\mathrm{PhD}$ dissertation, Columbia University, at http://digitalcommons.libraries.columbia.edu/dissertations/AAI3005712/, accessed April 2006. 
I. Juvina and E. Herder, 2004. "Lostness prediction using navigation styles," paper submitted to CHI2004 Conference, at http://www.cs.uu.nl/education/stage/interneprojecten/outlineresearch.pdf, accessed 15 March 2006.

I. Juvina and H. van Oostendorp, 2004. "Individual differences and behavioral aspects involved in modeling Web navigation," Lecture Notes In Computer Science, volume 3196, pp. 77-95, and at http://www.cs.uu.nl/people/ion/JuvinaAndVanOostendorpUAIS2004.pdf, accessed 15 March 2006.

N. Ford, D. Miller and N. Moss, 2001. "The role of individual differences in Internet searching: An empirical study," Journal Of The American Society For Information Science And Technology, volume 52, number 12, pp. 1049-1066.

M. Gui, 2006. "Disuguaglianze in Rete. Teoria della disuguaglianza digitale e analisi sperimentale delle capacità di reperimento di informazioni su Internet," $\mathrm{PhD}$ dissertation, University of MilanoBicocca, defended on 30 May 2006.

E. Hargittai and S. Shafer, 2006. "Differences in actual and perceived online skills: The role of gender," Social Science Quarterly, volume 87, number 2, pp. 432-448, and at http://www.eszter.com/research/pubs/hargittai-shafer-genderskills.pdf, accessed 20 April 2006.

E. Hargittai, 2004a. "Internet access and use in context," New Media and Society, volume 6, number 1, pp. 137-143.

E. Hargittai 2004b, "Classifying and coding online actions," Social Science Computer Review, volume 22, number 2, pp. 210-227.

E. Hargittai, 2002. "Second level digital divide: Differences in people's online skills," First Monday, volume 7, number 4 (April), at http://www.firstmonday.org/issues/issue7_4/hargittai/, accessed 27 August 2007.

K.S. Kim and B. Allen, 2001. "Person and context in information seeking: Interactions between cognitive and task variables," New Review of Information Behaviour Research, volume 2, pp. 1-16.

Lazonder, H.J. Biemans and I. Wopereis, 2000. "Differences between novice and experienced users in searching information on the World Wide Web," Journal of the American Society for Information Science, volume 51, number 6, pp. 576-581.

S. Liff and A. Shepherd, 2005. "An evolving gender digital divide?" Oxford Internet Institute, Internet Issue Brief, number 2 (July), at http://www.oii.ox.ac.uk/resources/publications/IB2all.pdf, accessed 15 March 2006. 
P. Norris, 2001. Digital divide: Civic engagement, information poverty, and the Internet worldwide. Cambridge: Cambridge University Press.

R.A. Palmquist and K.S. Kim, 2000. "Cognitive style and on-line database search experience as predictors of Web search performance," Journal of the American Society for Information Science, volume 51, number 6, pp. 558-566.

H.W. Park, 2002. "The digital divide in South Korea: Closing and widening divides in the 1990s," Electronic Journal of Communication, volume 12, numbers 1 and 2 , at http://www.cios.org/www/ejc/v12n102.htm.

J. Steyaert, 2000. "Digitale vaardigheden: Geletterdheid in de informatiesamenleving" ["Digital skills: Literacy in the information society"], at http://www.steyaert.org/Jan/Publicaties/2000.digitalskills.pdf, Rathenau Instituut, The Hague, Netherland.

J. van Dijk, 2006. "From digital divide to social opportunities," at http://www.gw.utwente.nl/vandijk/news/international_conference_for_b/051214_from_digital_divi de to.doc/.

J. van Dijk, 2005. The deepening divide: Inequality in the information society. London: Sage.

J. van Dijk, 2000. "Widening information gaps and policies of prevention," In: K. Hacker andf J. van Dijk (editors). Digital democracy: Issues of theory and practice. London: Sage, pp. 166-183.

J. van Dijk and K. Hacker, 2003. "The digital divide as a complex and dynamic phenomenon," Information Society, volume 19, number 4, pp. 315-326.

P. Wang, W.B. Hawk and C. Tenopir, 2000. "Users' interaction with World Wide Web resources: An exploratory study using a holistic approach," Information Processing and Management, volume 36, number 2, pp. 229-251.

M. Warschauer, 2002. "Reconceptualizing the digital divide," First Monday, volume 7, number 7 (July), at http://www.firstmonday.org/issues/issue7_7/warschauer/, accessed 15 March 2006.

M.D. White and M. Iivonen, 2001. "Questions as a factor in Web search strategy," Information Processing and Management, volume 37, number 5, pp. 721-740. 
M.D. White and M. Iivonen, 1999. "Factors influencing Web search strategies," paper presented at ASIS Annual Conference Washington, D.C., at http://www.glue.umd.edu/ whitemd/ASIS_papp.pdf, accessed March 2006. 